\title{
Study of the vortex-induced pressure excitation source in a Francis turbine draft tube by particle image velocimetry
}

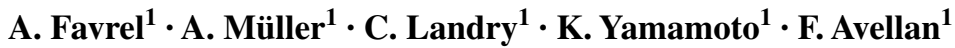

Received: 4 August 2015 / Accepted: 9 November 2015 / Published online: 26 November 2015

(C) Springer-Verlag Berlin Heidelberg 2015

\begin{abstract}
Francis turbines operating at part-load experience the development of a precessing cavitation vortex rope at the runner outlet, which acts as an excitation source for the hydraulic system. In case of resonance, the resulting pressure pulsations seriously compromise the stability of the machine and of the electrical grid to which it is connected. As such off-design conditions are increasingly required for the integration of unsteady renewable energy sources into the existing power system, an accurate assessment of the hydropower plant stability is crucial. However, the physical mechanisms driving this excitation source remain largely unclear. It is for instance essential to establish the link between the draft tube flow characteristics and the intensity of the excitation source. In this study, a twocomponent particle image velocimetry system is used to investigate the flow field at the runner outlet of a reducedscale physical model of a Francis turbine. The discharge value is varied from 55 to $81 \%$ of the value at the best efficiency point. A particular set-up is designed to guarantee a proper optical access across the complex geometry of the draft tube elbow. Based on phase-averaged velocity fields, the evolution of the vortex parameters with the discharge, such as the trajectory and the circulation, is determined for the first time. It is shown that the rise in the excitation source intensity is induced by an enlargement of the vortex trajectory and a simultaneous increase in the precession frequency, as well as the vortex circulation. Below a certain value of discharge, the structure of the vortex abruptly
\end{abstract}

A. Favrel

arthur.favrel@epfl.ch

1 Laboratory for Hydraulic Machines, Ecole Polytechnique Fédérale de Lausanne, Av. de Cour 33 bis, 1007 Lausanne, Switzerland changes and loses its coherence, leading to a drastic reduction in the intensity of the induced excitation source.

\section{Introduction}

Beyond a critical degree of swirl, rotating flows experience a hydrodynamic phenomenon, commonly called vortex breakdown. It is characterized by a sudden change in the flow structure with the formation of a stagnant point and a recirculation zone (Lucca-Negro and O'Doherty 2001). Under certain conditions, the development of a vortical structure precessing around the zone of recirculation may be observed (Gupta et al. 1984). This phenomenon, referred to as the precessing vortex core (PVC), is encountered in a wide range of engineering applications, leading to the production of an abundant literature reporting experimental and theoretical investigations (see Escudier 1987 and Syred 2006 for a review).

In the case of Francis turbines operating with a discharge lower than the nominal discharge (part-load condition), the vortex breakdown arises immediately at the runner outlet and produces a precessing helical vortex core in the draft tube. Its rotational frequency lies between 0.2 and 0.4 times the runner frequency (Nishi et al. 1982). The vortex precession in the draft tube acts as an excitation source for the hydromechanical system, inducing the propagation of pressure fluctuations. In case of resonance, pressure pulsations of high amplitude occur in the draft tube, which may put at risk the stability of the power plant (Rheingans 1940). As such off-design operations are increasingly required for the large-scale integration of intermittent energy sources into the grid, such as solar and wind, an accurate assessment of the stability of hydropower plants over a broad operating range is essential. This led to the development 
of one-dimensional flow models, where the contributions of Dörfler (1982), Couston and Philibert (1998), and more recently Alligné et al. (2014) have to be cited. Generally, the cavitation draft tube flow is modelled by a cavitation compliance parameter according to the definition introduced by Brennen and Acosta (1976), while the excitation source is represented by a source term in the momentum equation.

These models however require experimental validation. Particle image velocimetry is commonly used for the investigation of flow instabilities in hydraulic machines, from pump-turbines (Guggenberger et al. 2014; Widmer et al. 2011) to Francis turbines. In case of the latter, the axial velocity field in a vertical section of the draft tube cone was recently measured both at part-load (Iliescu et al. 2008; Kirschner et al. 2012) and at full-load conditions (Müller et al. 2013). From an analytical point of view, Susan-Resiga et al. (2006) modelled the mean swirling flow at the runner outlet by three elementary vortices, whose parameters are identified on the basis of time-averaged velocity profiles obtained experimentally. Kuibin et al. (2013) derived an analytical model recovering the time-averaged velocity profiles and the precession frequency. However, despite the large quantity of research dealing with the part-load issue (see Nishi and Liu 2013 for a review), the exact physical mechanisms driving the excitation source remain unclear and sparsely documented. A better understanding of this aspect is an important step towards an accurate prediction of pressure fluctuations and their transposition from the model to the prototype scale, which represents the ultimate challenge.

The present work aims at investigating the influence of the discharge on the vortex rope parameters and structure, and at highlighting how the intensity of the excitation source is linked to the vortex rope dynamics. The tangential flow field is investigated at the outlet of a reduced-scale physical model of a Francis turbine by means of a two-component PIV system. Measurements are taken in two different horizontal cross sections of the draft tube cone. The unsteady wall pressure is measured in order to describe the evolution of both the excitation frequency and the amplitude of pressure pulsations with the discharge. The measurements are taken in cavitation-free conditions in order to exclude the influence of parameters related to the presence of a cavitation volume. The latter drastically reduces the natural frequency of the system (Landry et al. 2014), which results in the risk of resonance (Favrel et al. 2014). Moreover, the presence of cavitation in the vortex core is prone to modify the vortex dynamics and consequently to influence the excitation source.
The test case is presented in Sect. 3, together with the evolution of both the excitation frequency and the amplitude of the pressure pulsations with the discharge, leading to the identification of three different flow regimes. The set-up for the PIV measurements is presented in Sect. 4, along with the methodology for calculating the evolution of the velocity fields over one precession cycle. The identification of both the vortex core limits and the vortex centre trajectory is also included. The behaviour of the precessing vortex core parameters as a function of the discharge is presented in Sect. 5. The used methodology and the physical mechanisms inducing the excitation source are finally discussed in Sect. 6.

\section{Background}

If the pressure level in the draft tube is decreased, the cavitation development is promoted and the vortex rope appears. A visualization is presented in Fig. 1a for the present test case. The periodical precession of this vortex induces the propagation of pressure fluctuations in the hydraulic system at the precession frequency. In the draft tube cone, these pressure fluctuations can be decomposed into two distinct components, the convective one and the synchronous one (Nishi et al. 1982). The convective component is a local pressure fluctuation induced by the rotation of the pressure field with the vortex. It is directly related to the local convection of the precessing vortex core in the draft tube cone. The synchronous component has an equal phase and amplitude for pressure sensors located in the same cross section of the cone and is able to propagate into the entire hydraulic system. Dörfler (1982) identified this component as the result of the excitation source induced by the vortex precession in the draft tube elbow. In cavitation conditions, the presence of a gaseous volume causes a significant drop of the wave speed in the draft tube and therefore of the system's eigenfrequencies. When the first eigenfrequency approaches the precession frequency with a growing cavitation volume, the synchronous pressure component becomes dominant and the system can enter into resonance, as illustrated in Fig. $1 b$.

However, in cavitation-free conditions, it is assumed that the first eigenfrequency of the system is sufficiently high to avoid any amplification of the synchronous component. Therefore, the evolution of both the excitation source intensity and the excitation frequency with the discharge value can be quantified in cavitation-free conditions by measuring pressure fluctuations directly in the feeding pipe, where they are purely synchronous. 



(a)

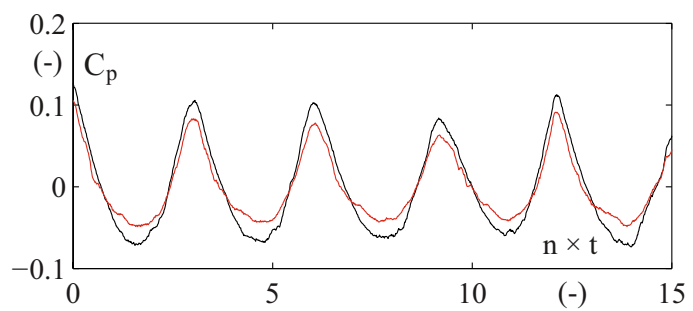

(b)

Fig. 1 Visualization of the cavitation precessing vortex rope together with two pressure signals measured in the same cross section of the draft tube cone in resonance conditions. a Visualization of the cavitation vortex rope. $\mathbf{b}$ Wall pressure signals measured in the cone

\section{Test case}

\subsection{Reduced-scale model}

The test case is a 1:16 reduced-scale physical model of a Francis turbine with a specific speed of $v=0.27$. It is installed on a closed-loop test rig of the EPFL Laboratory for Hydraulic Machines (see Fig. 2). The facility includes two axial doublevolute pumps generating the specified head. The value of the discharge is adjusted through the guide vanes opening of the turbine model. The cone of the draft tube is made of Plexiglas, providing an optical access for the laser sheet of the PIV system. Flush-mounted piezo-resistive pressure transducers are installed to measure the absolute wall pressure at different locations of the test rig. Two pairs of sensors are installed in two different cross sections of the draft tube cone. The pressure sensors located in the same cross section are separated with an angle equal to $\pi$. In addition, one sensor is installed in the feeding pipe to evaluate the amplitude of the synchronous pressure component independently of the convective component observed in the draft tube cone.

\subsection{Operating conditions}

Operating conditions in hydraulic machines are characterized by two non-dimensional parameters, the speed factor $n_{\mathrm{ED}}$ and the discharge factor $Q_{\mathrm{ED}}$ which are defined according to IEC Standards (1999) by:

$n_{\mathrm{ED}}=\frac{n D}{\sqrt{E}}$

$Q_{\mathrm{ED}}=\frac{Q}{D^{2} \sqrt{E}}$

where $n$ is the runner frequency, $Q$ is the discharge, $D$ is the runner outlet diameter, and $E$ is the specific hydraulic energy. In the present investigation, the speed factor is kept constant at the rated value of $n_{\mathrm{ED}}=0.288$, which corresponds to the value at the best efficiency point (BEP). The runner frequency $n$ and the hydraulic specific energy $E=g H$ are kept constant at the values $n=13.33 \mathrm{~Hz}$ and $E=263 \mathrm{~J} \mathrm{~kg}^{-1}$, respectively. Pressure measurements are first taken in a wide range of operating conditions in cavitation-free conditions by modifying step by step the discharge from 50 to $100 \%$ of the discharge value $Q_{0}$ at the BEP, in order to investigate the influence of the discharge on both the precession frequency and the amplitude of the synchronous pressure pulsations.

\subsection{Pressure fluctuations related to different flow regimes}

In cavitation-free conditions, pressure oscillations at a distinct frequency equal to about 0.25 times the runner frequency are observed for discharge values lower than $85 \%$ of the value at the BEP. This indicates the development of a precessing vortex rope in the draft tube. For discharge values between 50 and $85 \%$ of the value $Q_{0}$, the precession frequency $f_{\mathrm{PVC}}$ is determined by computing the auto-spectral density function of the pressure signal measured in the feeding pipe. Its evolution with the discharge is presented in Fig. 3a. The corresponding amplitude $G_{x x}\left(f_{\mathrm{PVC}}\right)$ of the auto-spectra is also determined, and its evolution with the discharge is plotted in Fig. 3b.

The results presented in Fig. 3 suggest that three different flow regimes occur in the range of the investigated operating 


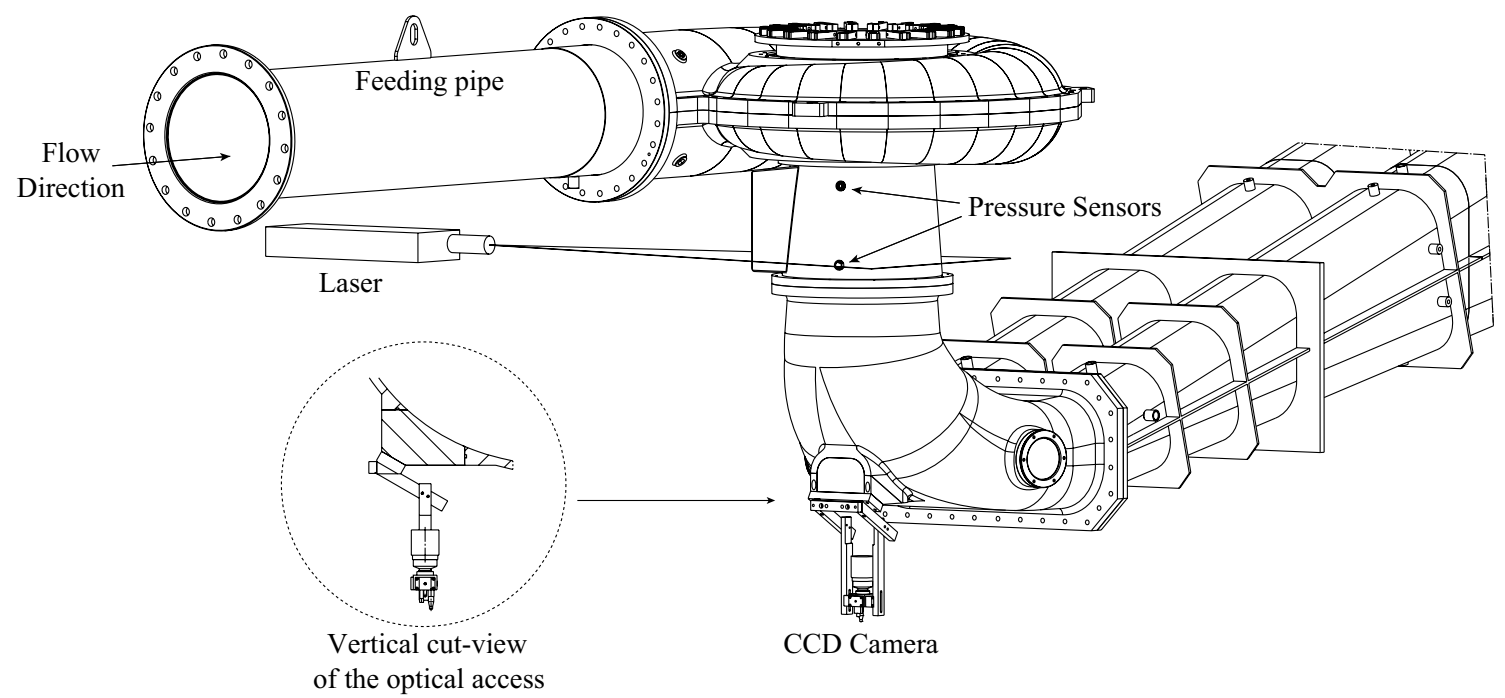

Fig. 2 Reduced-scale physical model of the Francis turbine installed on EPFL test rig PF3, with the set-up for the PIV measurements and a vertical cut-view of the camera support frame and the optical access



(a)

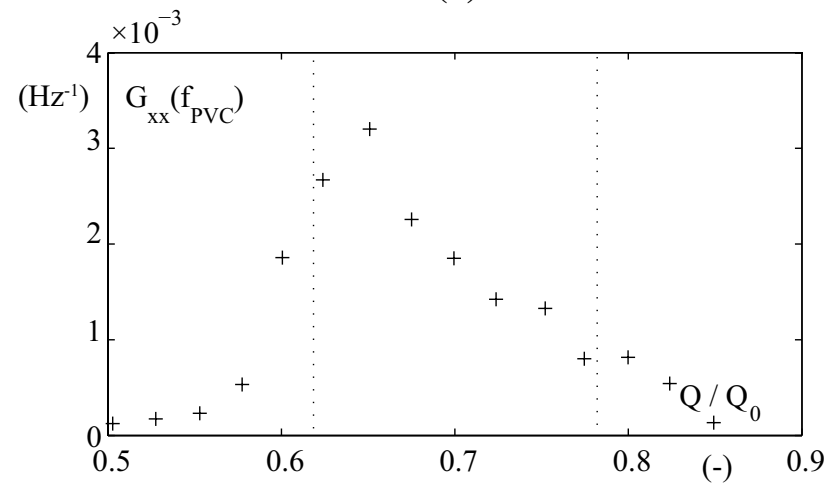

(b)

Fig. 3 a Precession frequency $f_{\mathrm{PVC}}$ made non-dimensional by the runner frequency $n$ and $\mathbf{b}$ auto-spectral amplitude at $f_{\mathrm{PVC}}$ for a pressure signal measured in the feeding pipe as a function of the discharge. The pressure signal and the discharge are made dimensionless by the turbine-specific head and the discharge value $Q_{0}$, respectively conditions. Within the first regime, from $Q=78$ to $85 \%$ of the value $Q_{0}$, the precession frequency remains quasi-constant and equal to about 0.26 times the runner frequency. The amplitude of the synchronous pressure pulsations at this frequency increases as the discharge decreases but remains low, indicating a weak hydroacoustic excitation. From 62 to $78 \%$ of the BEP, the precession frequency increases linearly from 0.26 to 0.34 times the runner frequency as the discharge decreases. In parallel, the amplitude of the pressure fluctuations significantly increases and reaches its maximum for a discharge value equal to $Q=0.65 \times Q_{0}$, which indicates a significant rise in the excitation source intensity in this segment. Below a discharge value of $Q=0.62 \times Q_{0}$, the results suggest that the dynamics of the precessing vortex rope are drastically modified. The precession frequency does not follow a particular law anymore, and the amplitude of the pressure fluctuations swiftly decreases, which indicates a significant reduction in the excitation source intensity.

A comparison between the cross spectrum of two pressure signals measured in the same cross section of the cone at two different discharge values, $Q=0.64 \times Q_{0}$ (Regime 2) and $Q=0.55 \times Q_{0}$ (Regime 3), is presented in Fig. 4 . The cross spectrum obtained at $Q=0.64 \times Q_{0}$ presents a very distinguishable peak at a low frequency, corresponding to the precession frequency. This type of spectrum is obtained for all discharge values between 62 and $85 \%$ of the value at the BEP. The spectrum at $Q=0.55 \times Q_{0}$ displays a similar qualitative shape, while being shifted to the right on the frequency axis and to the top regarding the mean amplitude level. The energy at the precession 


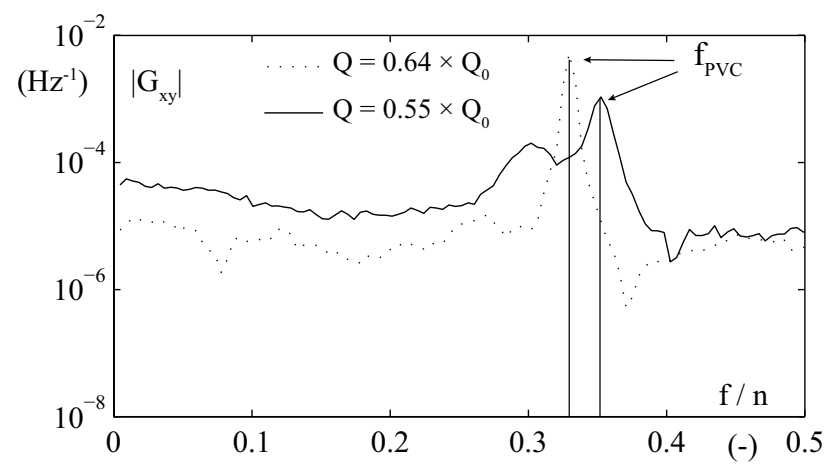

(a)

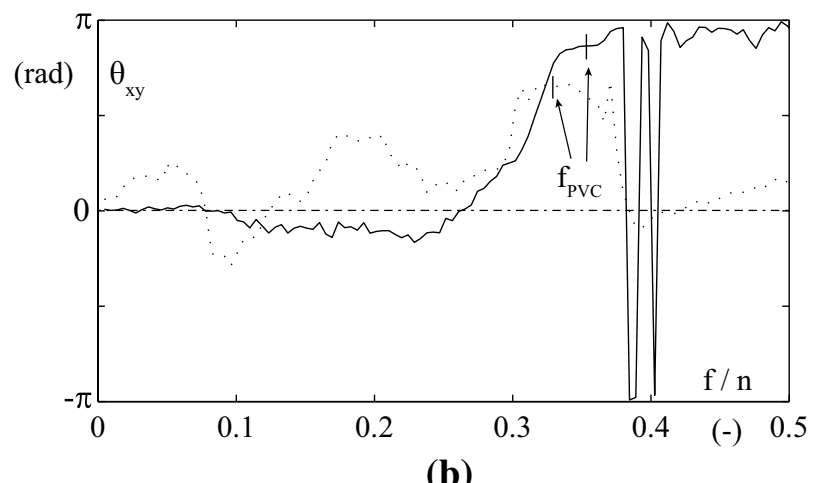

Fig. 4 a Amplitude and $\mathbf{b}$ phase of the cross-spectral density function of two pressure signals for the discharge values $Q=0.64 \times Q_{0}$ (dotted line) and $Q=0.55 \times Q_{0}$ (solid line). The frequency and the pressure signals are made dimensionless by the runner frequency and the turbine-specific head, respectively. The pressure sensors are located in the same cross section of the cone and are separated by an angle equal to $\pi$

frequency of 0.35 times the runner frequency is however spread over a broader frequency range, indicating a loss of periodicity in the pressure signal and therefore in the flow structure. At the sight of these observations, it is not possible anymore to extract a well-defined periodical behaviour in the velocity fields within regime 3 .

\section{Acquisition and analysis of PIV data}

\subsection{Experimental set-up}

The flow field is investigated in the draft tube cone by means of PIV performed in two horizontal cross sections, situated $0.39 \times D$ and $1.02 \times D$ downstream the runner outlet (Fig. 5), $D$ being the exit diameter of the runner. The diameters of the measurement sections are, respectively, equal to $1.05 \times D$ and $1.14 \times D$. The light sheet of 2-mm thickness is generated by a double-pulsed Nd:YAG laser and a cylindrical lens. The time interval between two consecutive pulses is adjusted at each operating point



Fig. 5 Vertical cut-view of the reduced-scale model, with the streamwise position of the PIV measurement sections. The used coordinate system is indicated by the unit vectors $x, y$, and $z$

to limit the out-of-plane particle displacement to $1 / 10$ of the laser sheet thickness (75 $\mu$ s for a discharge equal to $Q=0.320 \mathrm{~m}^{3} \mathrm{~s}^{-1}$, corresponding to an average axial velocity $C m=2.57 \mathrm{~m} \mathrm{~s}^{-1}$ in the Section 2). A waterbox is used to minimize the optical deformation of the laser sheet induced by the inclined wall of the cone.

The images are recorded using a CCD camera placed perpendicular to the laser sheet at the bottom of the draft tube elbow. The characteristics of both the laser and the camera are given in Table 1. A curved Plexiglas window fitting the shape of the draft tube is installed, providing an optical access for the camera. The camera is aligned with the coordinate system of the test rig (see Fig. 5) for a direct measurement of the velocity components $C x$ and $C y$ in this system. Due to the strong optical distortion introduced by the singular optical access, particular attention is paid to the calibration procedure. For each measurement section, a calibration image is taken with a circular dotted target covering the measurement section. The camera is fixed on a metal frame attached to the draft tube elbow, in order to preserve the relative position of the camera when the draft 
Table 1 Parameters of the PIV system

\begin{tabular}{ll} 
Laser & \\
Wavelength & $532 \mathrm{~nm}$ \\
Energy & $200 \mathrm{~mJ}$ \\
Type & $\begin{array}{l}\text { double-pulsed Nd:YAG laser } \\
\text { Model }\end{array}$ \\
Camera & \\
Resolution & $2048 \times 2048$ pixels \\
Lens & $60 \mathrm{~mm}$ \\
Filter & None \\
Model & Dantec FlowSense EO 4M \\
\hline
\end{tabular}

Table 2 List of investigated operating points

\begin{tabular}{lllll}
\hline Point & $Q_{\mathrm{ED}}(-)$ & $Q\left(\mathrm{~m}^{3} \mathrm{~s}^{-1}\right)$ & $Q / Q_{0}(-)$ & $\operatorname{Re}(-)$ \\
\hline 1 & 0.161 & 0.320 & 0.81 & $1.11 \times 10^{6}$ \\
2 & 0.155 & 0.308 & 0.78 & $1.07 \times 10^{6}$ \\
3 & 0.145 & 0.288 & 0.74 & $1.00 \times 10^{6}$ \\
4 & 0.135 & 0.268 & 0.68 & $0.93 \times 10^{6}$ \\
5 & 0.128 & 0.255 & 0.64 & $0.89 \times 10^{6}$ \\
6 & 0.120 & 0.239 & 0.60 & $0.83 \times 10^{6}$ \\
\hline
\end{tabular}

tube elbow is removed. As the measurements are taken in cavitation-free conditions, standard $20-\mu \mathrm{m}$ polyamide particles are used for seeding.

The measurements are taken at six different discharge values (see Table 2) for which an oscillation in both pressure and velocity signals can be observed, confirming the presence of a precessing vortex core. For a constant value of the speed factor $n_{\mathrm{ED}}$, a decrease in the discharge leads to a decrease in the axial velocity $C m_{\overline{1}}$ and an increase in the tangential velocity $C u_{\overline{1}}$ at the runner outlet. It results in an increase in the swirl rate of the flow leaving the runner, which is the driving parameter for the development of the precessing vortex rope. The swirl rate is defined as the ratio between the axial flux of angular momentum and the axial flux of axial momentum (Gupta et al. 1984). A modification of the discharge also alters the turbulence characteristics, represented by the Reynolds number defined as

$R e=\frac{C m_{\overline{1}} D}{v}=\frac{4 Q}{\pi D v}$

where $v$ is the kinematic viscosity and $C m_{1}$ the average axial velocity computed at the runner outlet. For all the investigated flow conditions, the Reynolds number is in the order of $10^{6}$, indicating a fully developed turbulent flow in the draft tube cone. As this value is relatively high, the precession frequency and the pressure fluctuation amplitude are assumed to be independent of viscous effects, as reported by Cassidy and Falvey (1970).

\subsection{Image post-processing}

Due to the nonlinear distortions induced by the complexly shaped optical access, each image from the camera is first dewarped using a third-order polynomial imaging model fit based on the calibration procedure described previously. Instantaneous velocity fields are then obtained by applying an adaptive cross-correlation method to each dewarped image pair. This method adapts the size and shape of the interrogation areas to local seeding densities and flow gradients. The minimum size for the interrogation area is set to $16 \times 16$ pixels. A universal adaptive outlier algorithm is applied to suppress the spurious vectors, which may result from reflections or the passage of gas bubbles through the laser sheet. The outlier velocity vectors are replaced by averaging the neighbouring vectors within a window of $5 \times 5$ velocity vectors. The resulting instantaneous velocity fields contain $272 \times 241$ vectors with a spatial resolution of $\Delta x=\Delta y=1.54 \mathrm{~mm}$ and $1.68 \mathrm{~mm}$ in the measurement sections 1 and 2 , respectively.

\subsection{Phase averaging procedure}

\subsubsection{Measurement procedure}

Different authors performed phase-locked PIV measurements by triggering the PIV acquisition directly with a reference pressure signal in order to recover the velocity field evolution over one typical period (Müller et al. 2013; Martinelli et al. 2012).

In the present study, the voltage of the internal trigger of the PIV system (Q-switch) is used to determine a unique time stamp for each recorded pair of images, which later enables the phase averaging. Hence, each voltage peak in Fig. 6 corresponds to a PIV acquisition. For a given operating point, the PIV data are acquired continuously with a sampling frequency of $10 \mathrm{~Hz}$, while the Q-switch voltage and the pressure signals are recorded synchronously with a sampling frequency of $1000 \mathrm{~Hz}$. For each operating point, a total of 10,000 pairs of images are acquired. An example of signals is shown in Fig. 6 for a shortened sample.

The mean phase averaging of a continuously sampled pressure signal in the draft tube is described by Müller et al. (2016). A similar technique is applied here to the velocity fields by using a reference pressure signal measured in the draft tube cone, $0.39 \times D$ downstream the runner outlet. After applying a low-pass filter to the pressure signal, the different precession cycles are easily defined by the position of the local pressure minima. Each cycle, corresponding to a period of $2 \pi$, is then divided into 90 phase windows of $2 \pi / 90$ width $\left(4^{\circ}\right)$. The velocity fields measured inside a given phase window over the entire acquisition time, identified by the corresponding Q-switch 




Fig. 6 Reference pressure signal made dimensionless by the turbinespecific head (black solid line) and output voltage from Q-switch laser (red solid line) for the operating point $Q=0.64 \times Q_{0}$

voltage peaks, are then averaged together. This results in 90 mean phase-averaged velocity fields, representing the flow behaviour over one typical precession period. As the distribution of the instantaneous velocity fields in the different phase windows is random, the number of averaged velocity fields for a given phase window varies from 90 to 120 . However, it is verified that 90 velocity fields are sufficient to reach a statistical convergence of the velocity field. An example of one instantaneous velocity field together with the corresponding mean version, resulting from the averaging of 120 instantaneous velocity fields, is provided in Fig. 7. Only a fraction of the measurement section including the vortex centre is plotted here. It can be noticed that the position of the vortex centre in the instantaneous velocity field slightly differs from the one in the phase-averaged velocity field, which is explained by the irregularity of the vortex precession.

The phase averaging suppresses the random turbulent fluctuations and isolates the periodical component of the velocity fields induced by the vortex precession. In the case of LDV data measured in rotating machinery, Glas et al. (2000) proposed a statistical evaluation of the phase-averaged velocity values and the corresponding turbulent values computed in a phase window of width $\Delta \phi$. The confidence interval of these quantities is estimated for different averaging methods. For the present test case, the confidence interval of both velocity components is evaluated for a given phase window at the operating point $Q / Q_{0}=0.64$. For a position within the vortex core, the confidence intervals are at $C x \pm 0.18 \mathrm{~m} \mathrm{~s}^{-1}$ and $C y \pm 0.24 \mathrm{~m} \mathrm{~s}^{-1}$ with a confidence of $95 \%$ and a number of samples equal to $N=121$. For a position within the irrotational zone, they are $C x \pm 0.088 \mathrm{~m} \mathrm{~s}^{-1}$ and $C y \pm 0.16 \mathrm{~m} \mathrm{~s}^{-1}$.

\subsubsection{Fourier series decomposition}

A Fourier series decomposition is applied to each phaseaveraged velocity component, yielding their pure periodic part. For a given spatial position $(x, y)$, the Fourier series decomposition of the velocity component $C y$ is expressed by:

$C y(x, y)=\sum_{n=1}^{N} A_{n} \cos \left(2 \pi \cdot f_{\mathrm{PVC}} \cdot n \cdot t+\theta_{n}\right)+\overline{C y}(x, y)$

with $A_{n}$ and $\theta_{n}$ the amplitude and the phase of the $n$th harmonics, respectively, and $\overline{C y}(x, y)$ the time-averaged mean value of the velocity component $C y$ at the position $(x, y)$. Limiting the number of harmonics to $N=3$ is sufficient to neglect the contribution of the higher-order harmonics. A comparison between the phase-averaged results and those obtained by applying the truncated Fourier series decomposition is provided in Fig. 8 for the velocity component $C y$ at the position $(x, y)=(0,0)$ (Fig. 8a) and its profile along the $x$-axis (Fig. 8b). Martinelli et al. (2012) found similarly that the contribution of the higher harmonics is negligible by fitting the power density function of the velocity components with an analytical expression. The velocity fields obtained with this method are used to compute the vortex characteristics, as described in the following section.
Fig. 7 a Instantaneous individual velocity field and $\mathbf{b}$ corresponding phase-averaged velocity field for the phase window $\phi=4 \pi / 3$ at the operating point $Q=0.64 \times Q_{0}$

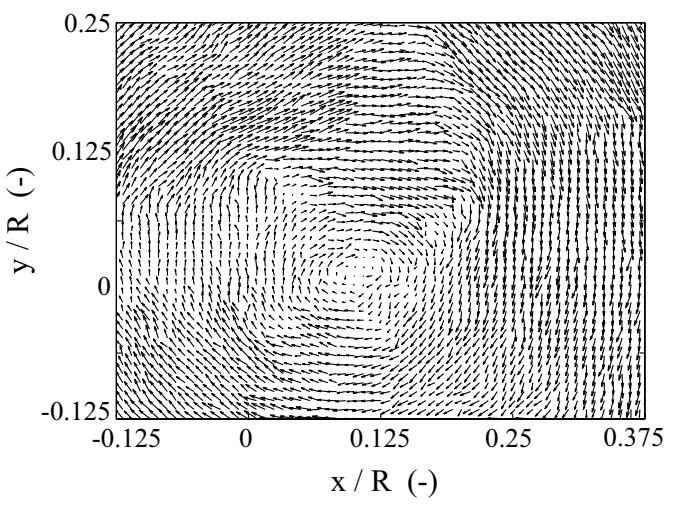

(a)

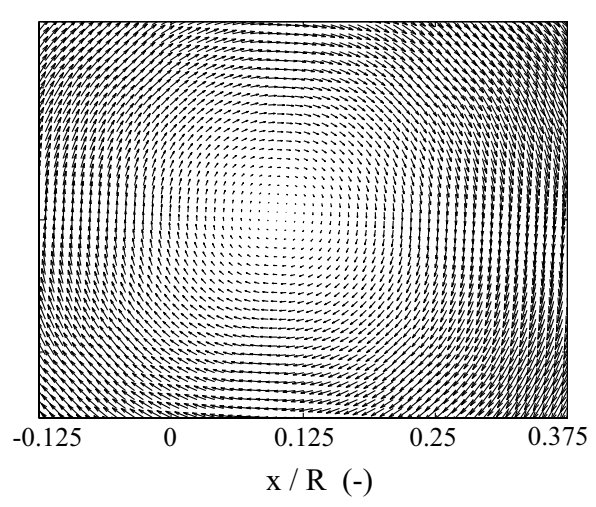

(b) 
Fig. 8 a Phase-averaged velocity component $C y$ over one precession cycle at the position $(x, y)=(0,0)$ and $\mathbf{b}$ profile of the phase-averaged velocity component $C y$ along the $x$-axis at the phase $\phi=\pi / 9$ for the operating point $Q=0.64 \times Q_{0}$. The velocity component $C y$ is made dimensionless by the outer runner velocity $U$

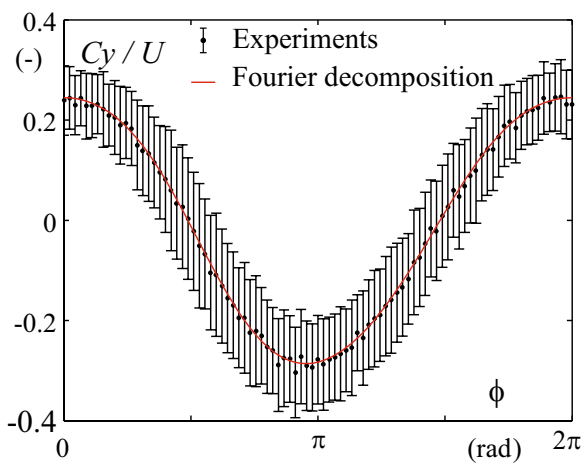

(a)

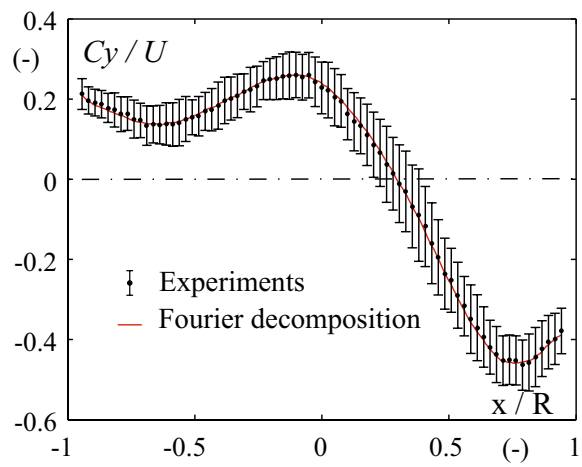

(b)

\subsection{Vortex parameters}

\subsubsection{Vortex centre}

The vortex centre can be identified by different techniques, which are mainly based on the computation of velocity gradients. For instance, Jeong and Hussain (1995) introduced the $\lambda_{2}$-criterion for the identification of vortical structures in turbulent flows. It has been used for vortex detection in the past (Cala et al. 2006; Martinelli et al. 2012). Graftieaux et al. (2001) proposed an algorithm to identify the vortex centre without computing velocity gradients, unlike the $\lambda_{2}$ -criterion-based techniques. They introduced a dimensionless scalar $\gamma_{1}$ defined at a given point $P$ by:

$\gamma_{1}(P)=\frac{1}{N} \sum_{\mathrm{S}} \frac{(\mathbf{P M} \wedge \mathbf{C}(M)) \cdot \mathbf{z}}{\|\mathbf{P M}\| \cdot\|\mathbf{C}(M)\|}$

with $S$ a rectangular domain of fixed size surrounding the point $P, M$ a point inside the domain $S$ and $N$ the number of points inside $S$. The symbols $\wedge$ and $\cdot$ correspond to the cross product and the scalar product, respectively. $\mathbf{C}(M)$ is the velocity vector in the $(x, y)$-plane and $\mathbf{z}$ the unit vector normal to the $(x, y)$-plan. Graftieaux et al. (2001) showed that in the case of an ideal axisymmetrical vortex, the sca$\operatorname{lar}\left|\gamma_{1}\right|$ reaches its maximum equal to 1 at the vortex centre. In a general case, the location of the vortex centre is identified as the position where the scalar $\left|\gamma_{1}\right|$ is at its maximum. Recently, this algorithm has been successfully implemented by Dreyer et al. (2014) for the detection of the tip leakage vortex centre and the correction of the vortex axis wandering. For the present investigation, the vortex centre is detected in the phase-averaged velocity fields and the instantaneous velocity fields, enabling a statistical analysis of the vortex position for the complete precession cycle. An example of distribution of the vortex centre position in the $(x, y)$-plane, together with the corresponding average trajectory of the vortex centre, is presented in Fig. 9. A dispersion of the instantaneous vortex centre around the average trajectory is observed, indicating a random component in the vortex precession.

\subsubsection{Vortex circulation}

The normal component of the vorticity $\omega_{z}=\frac{\partial C y}{\partial x}-\frac{\partial C x}{\partial y}$ is computed by direct derivation of the velocity components $(C x, C y)$, using a 5-point stencil numerical scheme. The spatial resolution of the velocity fields is first increased by applying a cubic spline interpolation in the $(x, y)$-plane for both velocity components. An example of the phase-averaged velocity field magnitude, together with the corresponding vorticity distribution, is presented in Fig. 10 for the operating point $Q=0.64 \times Q_{0}$. The velocity field is composed of two different zones: the vortex core, surrounding the vortex centre and characterized by a strong vorticity, and the surrounding zone in which the flow is quasi-irrotational.

The circulation $\Gamma$ of the vortex is equal to:

$\Gamma=\oint_{\mathscr{C}} \mathbf{C} \cdot \mathrm{d} \mathbf{l}=\int_{S} \omega_{z} \cdot \mathrm{d} S$

with $\mathscr{C}$ and $S$ the contour and the surface of the vortex core, respectively. The vortex circulation can be computed by a surface integration of the vorticity within the boundaries of the vortex core, or by a curvilinear integration of the tangential velocity along the vortex core limits. Accordingly, the limits of the vortex core must be defined properly. For this purpose, Graftieaux et al. (2001) proposed a second algorithm for the detection of the boundaries of large-scale vortices, which considers only the topology of the velocity field. They introduced another scalar $\gamma_{2}$ defined as:

$\gamma_{2}(P)=\frac{1}{N} \sum_{\mathrm{S}} \frac{(\mathbf{P M} \wedge(\mathbf{C}(M)-\mathbf{C}(P))) \cdot \mathbf{z}}{\|\mathbf{P M}\| \cdot\|\mathbf{C}(M)-\mathbf{C}(P)\|}$

with $\mathbf{C}(P)$ the average velocity vector within the domain $S$. Graftieaux et al. (2001) showed that when the scalar $\left|\gamma_{2}\right|$ is greater than $2 / \pi$, the flow is locally dominated by the rotating motion, corresponding to the vortex core zone. For each 


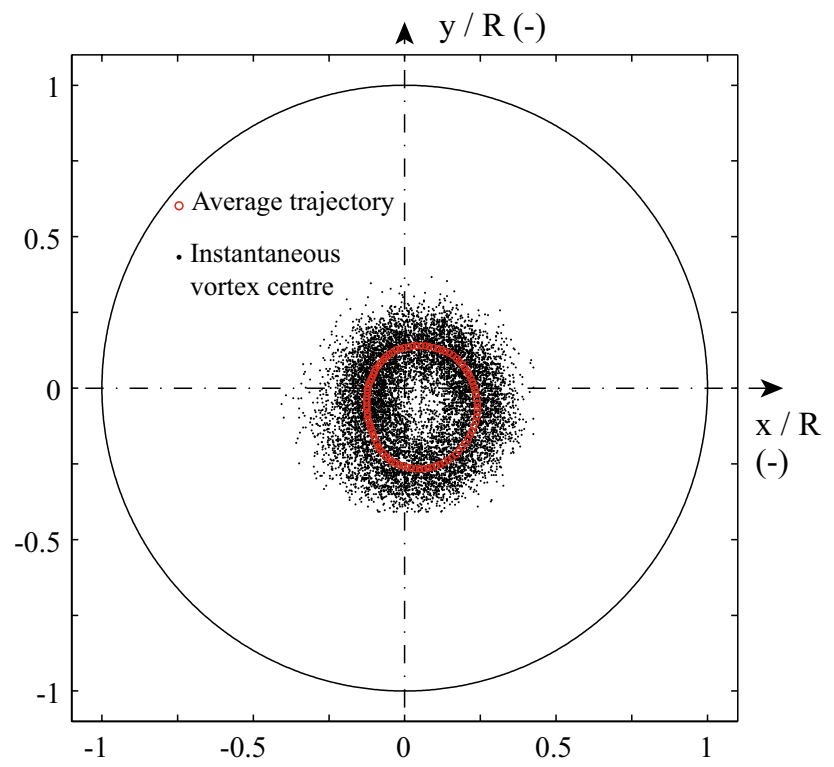

Fig. 9 Trajectory of the vortex centre position over one precession cycle for $Q=0.64 \times Q_{0}$. The black dots correspond to the vortex centre position determined for each instantaneous velocity field, and the red dots correspond to the average trajectory of the vortex centre determined with the phase-averaged velocity fields

operating point, the limits of the vortex core are determined for each phase of the precession period. The boundaries of the vortex core are made visible in Fig. 10 by the black solid contour. The vortex circulation is first computed by a surface integration of the vorticity. To verify the accuracy of this computation, the curvilinear integration of the tangential velocity is also implemented. Without further proof, the difference between both results does not exceed $0.15 \%$ of the value obtained by surface integration in the measurement section 1 and $0.2 \%$ in the measurement section 2 .

\section{Results}

\subsection{Vorticity and circulation}

The phase-averaged velocity fields are used to investigate the structure of the precessing vortex core for the different flow regimes introduced in Sect. 3. The magnitude $\sqrt{C x^{2}+C y^{2}}$ of the phase-averaged velocity field at a given point of the precession cycle is reported in Fig. 11 for each investigated discharge value. The presented phase corresponds to the instant for which the vortex centre (represented by the white crosses in Fig. 11) is situated exactly on the positive $x$-axis. The limits of the vortex core are drawn as a solid black contour. The corresponding vorticity distributions are presented in Fig. 12. For all the operating points between 81 and $60 \%$ of the discharge value at the BEP, a



(a)

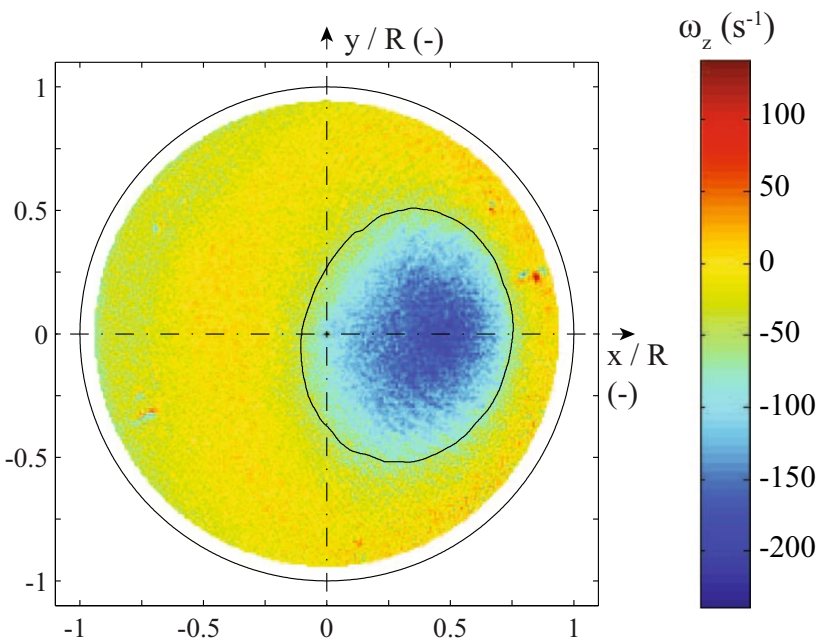

(b)

Fig. 10 a Velocity magnitude and $\mathbf{b}$ vorticity for a given phase of the precession cycle at the operating point $Q=0.64 \times Q_{0}$. The solid lines correspond to the limits of the vortex core

wide vortical structure is identifiable, whose core surface is greater than $19 \%$ of the total surface of the measurement section. In this range of discharge values, the identified vortical structure, corresponding to the precessing vortex core, is characterized by a high vorticity near the vortex centre and is surrounded by a zone of quasi-irrotational flow. However, varying the discharge changes the flow structure through the swirl rate, which is the driving parameter of the precessing vortex core. At a low swirl degree, with a discharge equal to $Q=0.81 \times Q_{0}$, the vortex core is quasicircular and the tangential velocity distribution around the vortex centre is nearly axisymmetric. A slight acceleration of the tangential flow between the vortex centre and the cone wall starts however to appear. For $Q=0.64 \times Q_{0}$, the vortex core is deformed and takes an elliptical shape. 


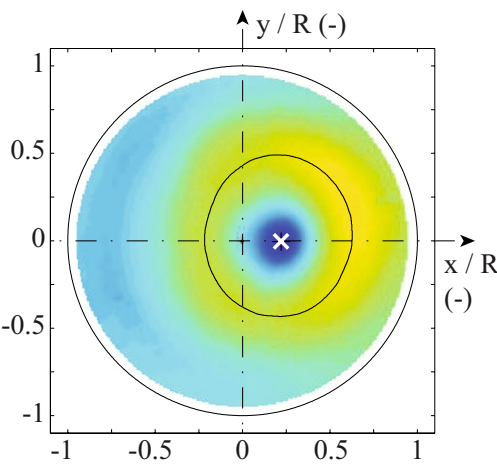

(a) $\mathrm{Q} / \mathrm{Q}_{0}=0.81$

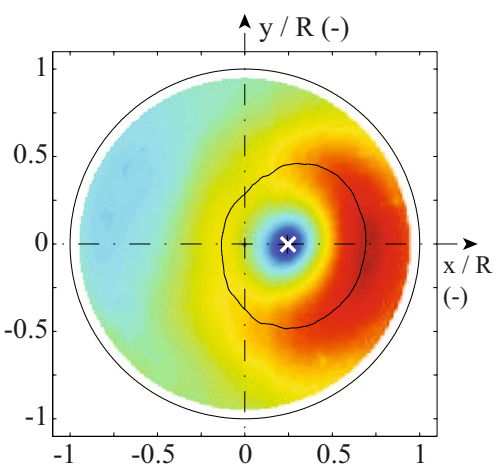

(d) Q / $\mathrm{Q}_{0}=0.68$

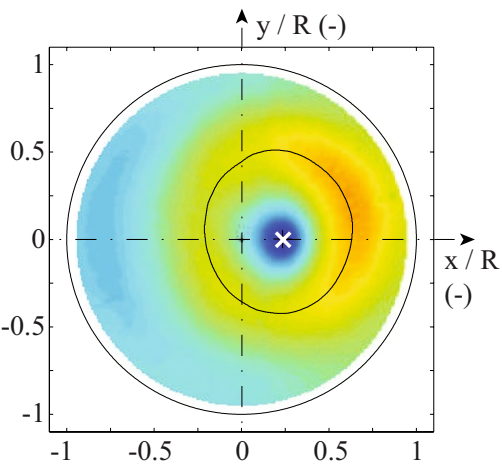

(b) $\mathrm{Q} / \mathrm{Q}_{0}=0.78$

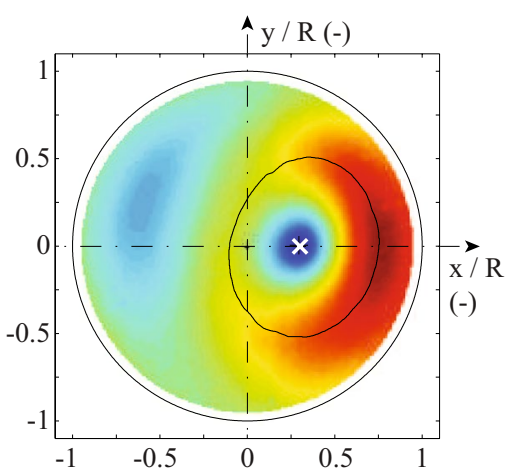

(e) Q / $\mathrm{Q}_{0}=0.64$

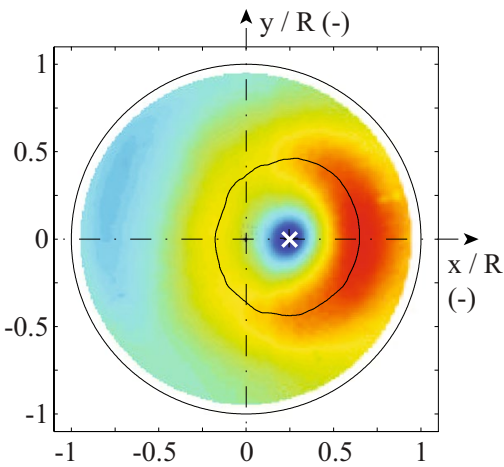

(c) $\mathrm{Q} / \mathrm{Q}_{0}=0.74$

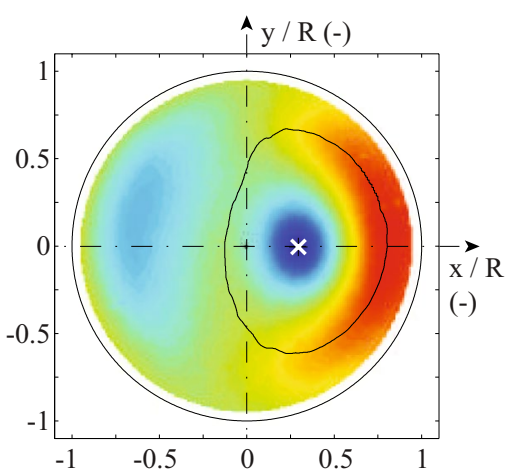

(f) $\mathrm{Q} / \mathrm{Q}_{0}=0.60$

Fig. 11 Velocity magnitude in the section 2 at a given phase for different discharge values. The black solid lines represent the limits of the vortex core, whereas the white crosses indicate the position of the vortex centre

The vorticity within the limit of the vortex core strongly increases for this point, as documented in Fig. 12e. Moreover, the velocity field around the vortex core is asymmetric, with a higher tangential velocity between the vortex centre and the cone wall. This phenomenon has already been reported by different authors (for instance Cala et al. 2006), and it was suggested that this effect is produced by the squeezing of the swirling flow between the vortex centre and the wall, as the angular momentum flux is conserved (Griffiths et al. 1998). The vortex centre moves closer to the cone wall when the discharge is decreased, inducing a more important contraction of the flow between the vortex centre and the solid boundary and consequently a more important acceleration of the flow in this zone.

The vortex circulation is computed at each phase value and then averaged over the complete precession period for all the investigated operating points. Its evolution with the discharge, together with the corresponding standard deviation, is reported in Fig. 13. A decrease in the vortex circulation away from the runner outlet in the direction of the flow is observed, which illustrates the gradual decay of the vortex strength due to viscous effects. However, the vortex circulation follows the same evolution in both measurement sections: It increases quasi-linearly when the discharge decreases. For discharge values $Q / Q_{0} \leq 0.6$, the flow fluctuations gradually lose their periodicity, causing a significant increase in the dispersion of the instantaneous vortex centres. Even though it is possible to plot the vortex cores in Figs. 11 and 12 for the case $Q=0.6 \times Q_{0}$, its limits are not well defined for certain phase values, and the algorithm for the calculation of the vortex circulation fails.

\subsection{Vortex trajectory}

The average trajectory of the vortex centre in all measurement sections is recovered by determining the vortex centre position at each phase of the precession cycle. The results for both sections 1 and 2 are presented in Fig. 14, each sub-figure representing the trajectory of the vortex centre for a given discharge. A strong asymmetry of the trajectories is observed, which is increasingly pronounced when lowering the discharge. The vortex centre precesses around an axis which differs slightly from the geometrical centre of the cross section. More precisely, the trajectory at $Q=0.81 \times Q_{0}$ and $Q=0.78 \times Q_{0}$, situated, respectively, in the regime 1 and just after the transition from regime 1 


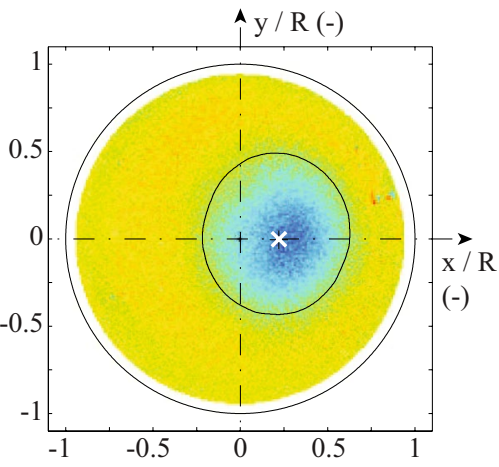

(a) $\mathrm{Q} / \mathrm{Q}_{0}=0.81$

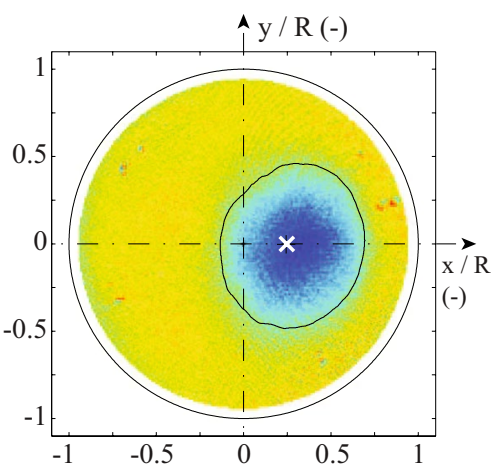

(d) $\mathrm{Q} / \mathrm{Q}_{0}=0.68$

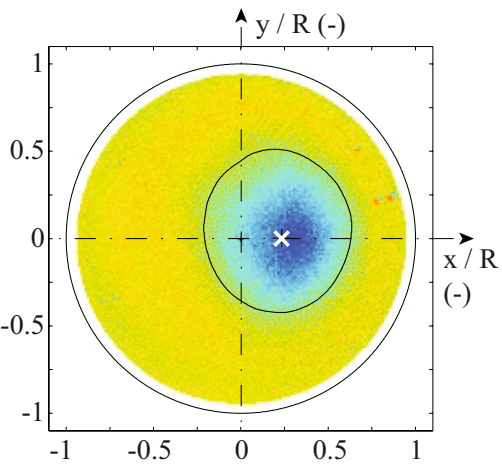

(b) $\mathrm{Q} / \mathrm{Q}_{0}=0.78$

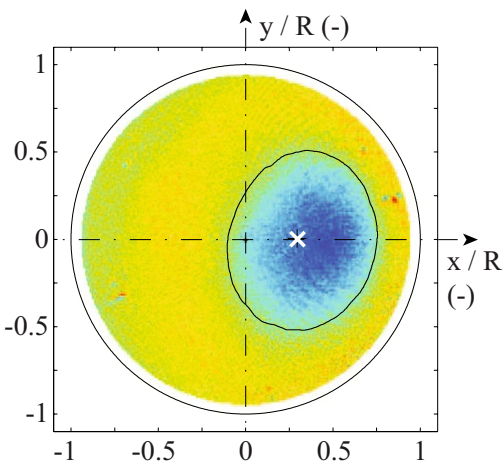

(e) $\mathrm{Q} / \mathrm{Q}_{0}=0.64$

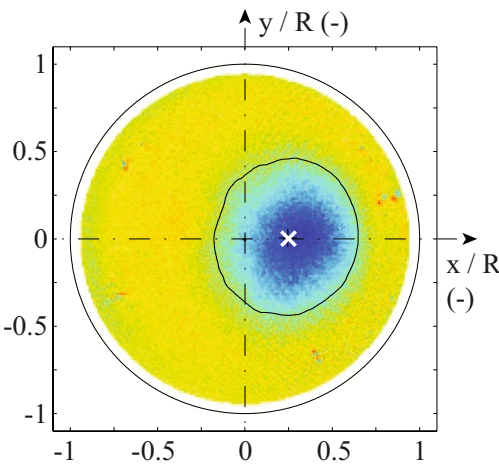

(c) $\mathrm{Q} / \mathrm{Q}_{0}=0.74$

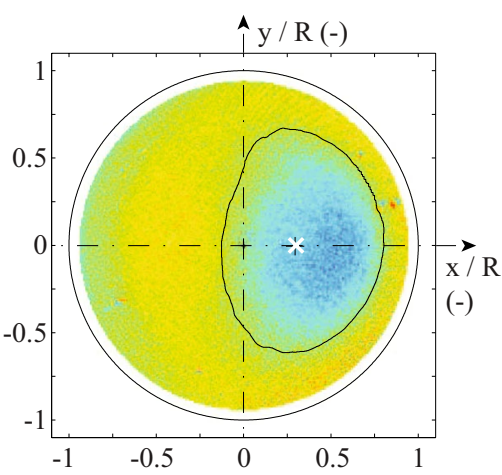

(f) $\mathrm{Q} / \mathrm{Q}_{0}=0.60$



$-100$

$-200$

Fig. 12 Normal component of the vorticity $w_{z}$ in the section 2 at a given phase for different discharge values. The black solid lines represent the limits of the vortex core, whereas the white crosses indicate the position of the vortex centre

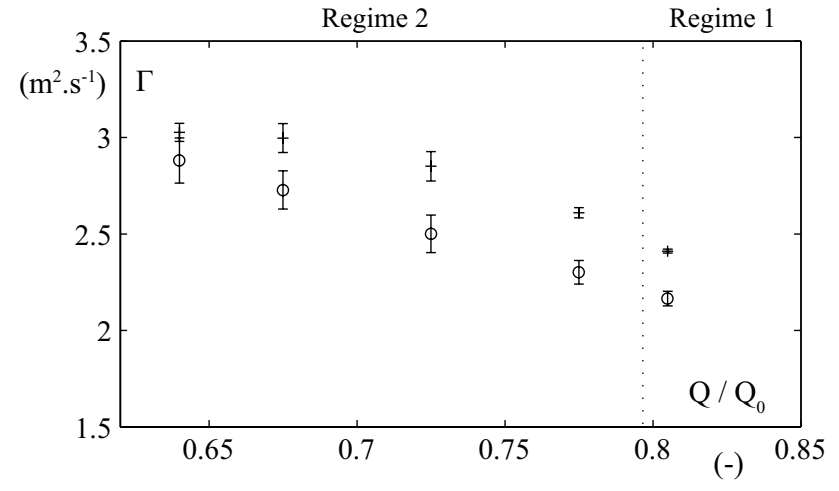

Fig. 13 Vortex circulation averaged over one precession cycle and its corresponding standard deviation as a function of the discharge. The markers + and $\circ$ represent the results obtained in section. 1 and 2 , respectively

to regime 2 , is quasi-circular and very similar, aside from a slight difference in their radius. This indicates that the vortex characteristics experience no significant change in regime 1 and only start altering from $Q=0.78 \times Q_{0}$ decreasing. In the flow regime 2 , from 78 to $62 \%$ of the $\mathrm{BEP}$, the trajectory of the vortex widens when the discharge decreases and the swirl degree leaving the runner increases. The length $l$ of the average vortex centre trajectory is computed for each value of the discharge by a curvilinear integral. Its evolution with the discharge is reported in Fig. 15 for both measurement sections 1 and 2. The relationship is linear in both sections, though the trajectory length is higher in the section 2, illustrating the widening of the vortex trajectory away from the runner outlet. The distance $l$ covered by the vortex centre over one precession period reaches its maximum in the section 2 at the operating point $Q=0.64 \times Q_{0}$, when the excitation source is also at its maximum. Moreover, the trajectory, initially quasi-circular and centred within the cross section at the point $Q=0.78 \times Q_{0}$, becomes increasingly asymmetrical, showing the growing influence of the elbow on the upstream flow when the swirl rate increases. This evolution is observed for all measurement sections, however with a more pronounced asymmetry in section 1 . After the transition from regime 2 to regime 3 , although the precession frequency does not diminish, the trajectory of the vortex retracts and becomes nearly circular and centred around the geometrical axis of the measurement sections, as illustrated by the trajectory obtained at the operating point 


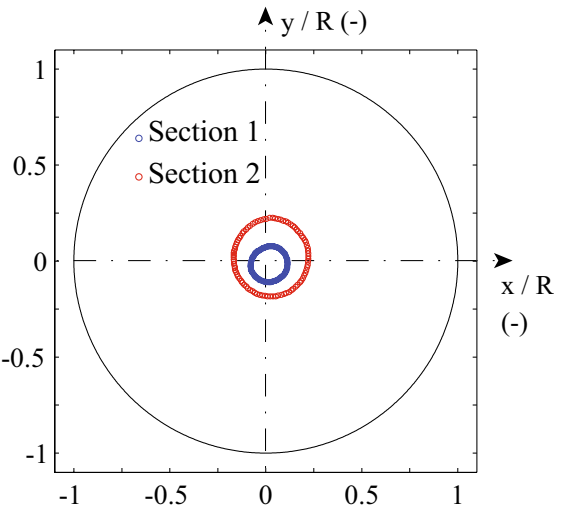

(a) $\mathrm{Q} / \mathrm{Q}_{0}=0.81$



(d) $\mathrm{Q} / \mathrm{Q}_{0}=0.68$

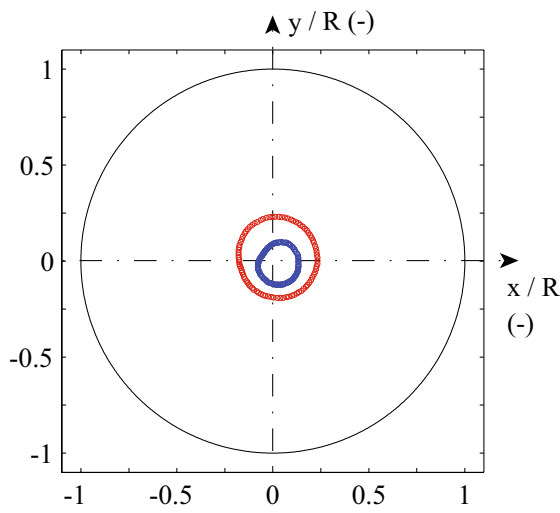

(b) $\mathrm{Q} / \mathrm{Q}_{0}=0.78$

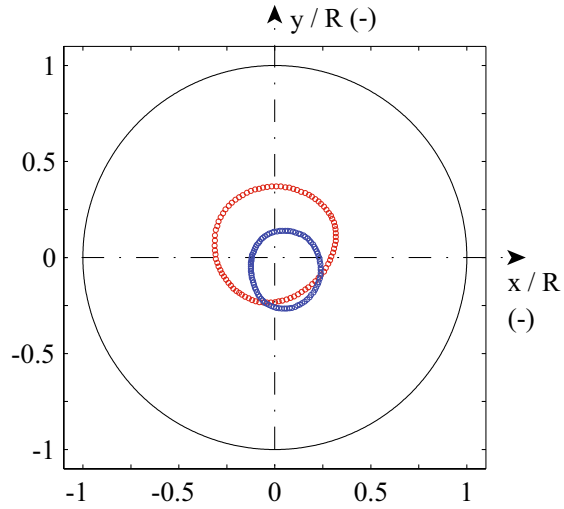

(e) $\mathrm{Q} / \mathrm{Q}_{0}=0.64$

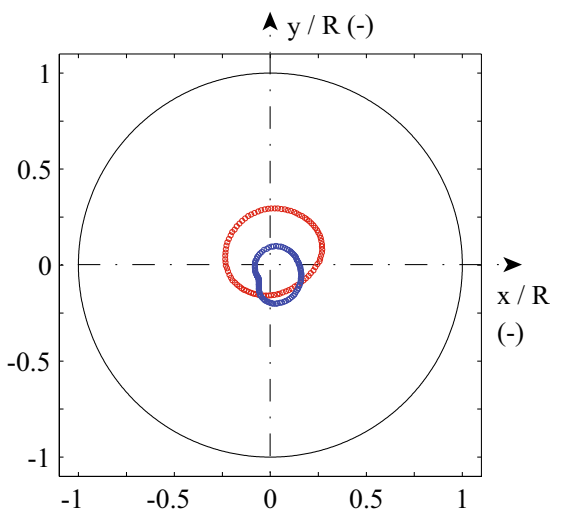

(c) $\mathrm{Q} / \mathrm{Q}_{0}=0.74$

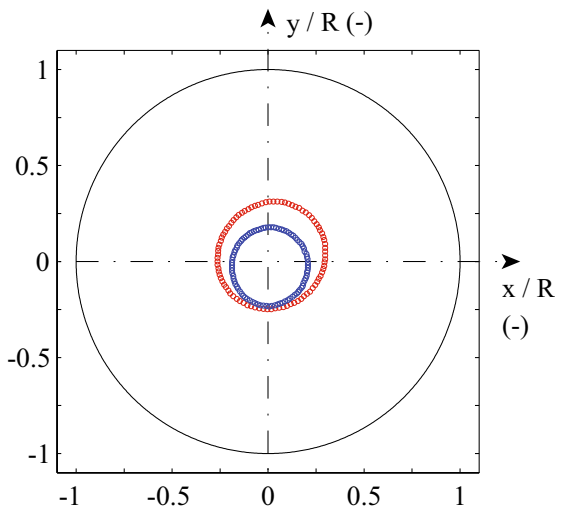

(f) $\mathrm{Q} / \mathrm{Q}_{0}=0.60$

Fig. 14 Vortex centre trajectory during one precession cycle for different discharge values in measurement sections 1 and 2

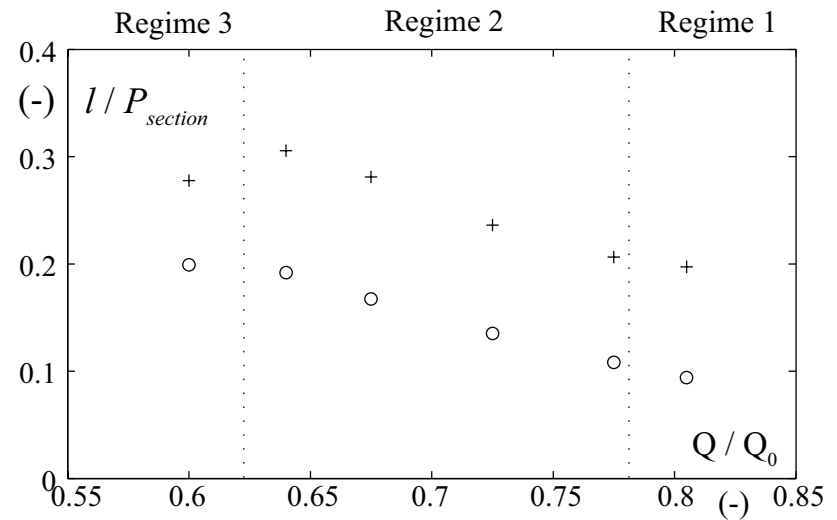

Fig. 15 Length $l$ of the average vortex centre trajectory as a function of the discharge in the sections $1(+)$ and $2(0)$. The length $l$ and the discharge are made dimensionless by the perimeter of the section and the discharge value $Q_{0}$ at the BEP, respectively

$Q=0.60 \times Q_{0}$. Beyond the transition between the regimes 2 and 3 , the distance $l$ covered by the vortex centre diminishes drastically in section 2 , whereas it remains stable in section 1.

\section{Discussion}

\subsection{Vortex centre dispersion}

As shown in Sect. 4.4.1, the instantaneous position of the vortex centre presents a dispersion around the average trajectory of the vortex. It is accompanied by a slight dispersion of the precession period from one cycle to another. The possible reasons for this are the random flow component induced by the fully developed turbulence and the potential fluctuations generated by the test rig control (runner and pump speeds).

The dispersion of the vortex centre for a given phase of the precession cycle is not supposed to affect the computation of the average vortex position and by extension the average vortex trajectory. However, it may introduce an artificial diffusion of the phase-averaged velocity fields. The vortex core size would consequently be overestimated, and the vortex circulation estimation would be biased. In the case of steady vortices, such as tip leakage vortices, a random fluctuation of the vortex centre is also observed and may be corrected by different techniques in order to 
avoid an overestimation of the vortex core (Dreyer et al. 2014; Bhagwat and Ramasamy 2012). In the case of a precessing vortex core, Ingvorsen et al. (2013) estimate the radial profile of the velocity components by averaging the instantaneous velocity fields centred on the instantaneous vortex centres. However, in the present test case, the use of this method is more complex due to the strong asymmetry of the vortex core observed in Fig. 11. Nevertheless, it is assumed that the phase averaging of the velocity fields mitigates this diffusion effect in regimes 1 and 2, where the average distance between the instantaneous vortex centres and the mean vortex centre is inferior to $15 \%$ of the vortex core size. The influence of the vortex position dispersion for a given phase will be subject of further investigations.

\subsection{Excitation mechanisms}

The previous results show that the increase in the swirl rate of the flow leaving the runner induces an increase in both the precession frequency and the vortex circulation, as well as an enlargement of the vortex trajectory. These effects are accompanied by a growth of the synchronous pressure pulsation amplitudes, which indicates an increase in the excitation source strength. It is therefore suggested that the latter is directly related to the vortex strength and the diameter of the vortex trajectory.

By comparing the pressure fluctuations induced by the precessing vortex core in elbowed and rectilinear draft tubes, Nishi et al. (1982) showed that the presence of the elbow is the main cause for the establishment of synchronous pressure pulsations. Later, Fanelli (1989) theoretically confirmed these experimental observations by representing the flow in the draft tube by the superposition of two potential-flow fields. The flow in the draft tube elbow is subject to separation at the intrados of the elbow, as observed in simple applications with elbowed pipes (Takamura et al. 2012). This flow separation zone, illustrated in Fig. 16, which impacts the pressure recovery in the draft tube, is altered by the presence of the vortex rope. The precession movement therefore introduces a periodical fluctuation of the pressure recovery $\tilde{p}_{\text {recov }}(t)$, whose amplitude is a function of the vortex circulation and trajectory (Fanelli 1989). This gives rise to the propagation of synchronous pressure pulsations, exciting the system at the precession frequency. A widening of the vortex core trajectory and a larger circulation hence result in a more significant variation in the separation zone and the pressure recovery, and as a consequence in an increase in the excitation source intensity.

Below a certain value of the discharge, the precessing vortex core loses its coherence, which is confirmed by the dispersion of the energy around the precession frequency in the auto-spectral density function of the pressure signals measured in the draft tube and the feeding pipe. As a

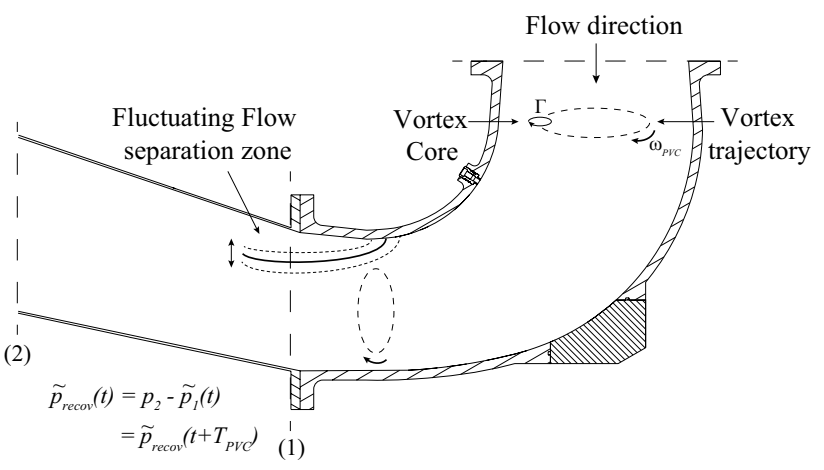

Fig. 16 Fluctuations of the flow separation zone induced by the precession of the vortex in the draft tube elbow

consequence, the precession of the vortex core ceases to induce coherent fluctuations of the pressure recovery at a well-defined frequency, and the resulting excitation of the hydromechanical system is suspended. It finally results in a sudden decrease in the amplitude of the synchronous pressure pulsations.

\section{Summary and conclusion}

The flow field at the outlet of a Francis turbine operating at part load is investigated with a two-component PIV system in cavitation-free conditions. The flow in the draft tube features the development of a precessing vortex core, which acts as an excitation source for the hydromechanical system. The PIV measurements are taken in two horizontal cross sections of the draft tube cone for different discharge values, covering a wide range of load conditions. Furthermore, pressure fluctuations are measured at different locations to highlight the influence of the discharge on both the vortex precession frequency and the amplitude of the synchronous pressure fluctuations induced by the excitation source. For each investigated flow condition, the periodical evolution of the velocity field over one mean precession cycle is recovered by phase averaging. The identification of the precession periods is based on a reference pressure signal measured in the draft tube cone. From the phaseaveraged velocity fields, the vortex centre position and the vortex core boundaries are identified, yielding the vortex trajectory during one precession cycle and the circulation within the vortex core for each measurement section.

The results highlight the existence of three different flow regimes depending on the discharge value, as well as a direct link between the vortex parameters and the amplitude of the synchronous pressure pulsations. From 78 to $85 \%$ of the discharge at the BEP, the precession frequency remains constant and then increases linearly as the discharge value is decreased from 62 to $78 \%$ of the value at the best 
efficiency point (BEP). This is accompanied by a linear increase in the vortex circulation and a broadening of the vortex trajectory. The modification of the vortex parameters results in an important increase in the amplitude of the synchronous pressure pulsations at the precession frequency, which is assumed to be a consequence of the increase in the excitation source intensity. It is suggested that a wider vortex trajectory, together with a higher value of its circulation, induces greater fluctuations of the pressure recovery in the diffuser due to the interaction of the main precessing vortex core with the flow separation zone formed at the elbow intrados. However, below a certain value of discharge, the vortex trajectory retracts, along with a sudden decrease in the synchronous pressure pulsations amplitude. The precessing vortex core abruptly loses its coherence, resulting in a weakening of the excitation source.

In summary, the amplitude of the synchronous pressure pulsations is strongly dependent on the trajectory, the strength and the coherence of the vortex, as they play a key role in the interaction of the precessing vortex core with the secondary flow in the elbow. As a consequence, special attention is to be paid to the elbow design to mitigate the establishment of a separation zone arising at the elbow intrados and to minimize the impact of the vortex precession on the pressure recovery in the diffuser. Further investigations will focus on the interaction between the main precessing vortex core and the secondary flow arising in the elbow by investigating the flow field at its outlet.

Acknowledgments The research leading to the results published in this paper is part of the HYPERBOLE Research Project, granted by the European Commission (ERC/FP7-ENERGY-2013-1-Grant 608532). The authors would also like to thank BC Hydro for making available the reduced-scale model, in particular Danny Burggraeve and Jacob Iosfin. Moreover, the authors would like to acknowledge the commitment of the Laboratory for Hydraulic Machines' technical staff, especially Georges Crittin, Maxime Raton, Victor Rivas, Alain Renaud, and Vincent Berruex.

\section{References}

Alligné S, Nicolet C, Tsujimoto Y, Avellan F (2014) Cavitation surge modelling in Francis turbine draft tube. J Hydraul Res 52(3):1-13

Bhagwat M, Ramasamy M (2012) Effect of tip vortex aperiodicity on measurement uncertainty. Exp Fluids 53(5):1191-1202

Brennen C, Acosta A (1976) Dynamic transfer function for a cavitating inducer. J Fluids Eng Trans ASME 98 Ser 1(2):182-191

Cala C, Fernandes E, Heitor M, Shtork S (2006) Coherent structures in unsteady swirling jet flow. Exp Fluids 40(2):267-276

Cassidy J, Falvey H (1970) Observations of unsteady flow arising after vortex breakdown. J Fluid Mech 41(pt 4):727-736

Couston M, Philibert R (1998) Partial load modelling of gaseous Francis turbine rope. Int J Hydropower Dams 1:146-158

Dörfler P (1982) System dynamics of the Francis turbine half load surge. In: Proceedings of the 11th IAHR symposium on operating problem of pump stations and powerplants, Amsterdam, Netherlands

Dreyer M, Decaix J, Münch-Alligné C, Farhat M (2014) Mind the gap: a new insight into the tip leakage vortex using stereo-PIV. Exp Fluids 55(11):1-13

Escudier M (1987) Confined vortices in flow machinery. Annu Rev Fluid Mech 19:27-52

Fanelli M (1989) Vortex rope in the draft tube of Francis turbines operating at partial load. A proposal for a mathematical model. J Hydraul Res 27(6):769-807

Favrel A, Landry C, Müller A, Yamamoto K, Avellan F (2014) Hydroacoustic resonance behavior in presence of a precessing vortex rope: observation of a lock-in phenomenon at part load Francis turbine operation. IOP conference series: earth and environmental science, 22

Glas W, Forstner M, Kuhn K, Jaberg H (2000) Smoothing and statistical evaluation of laser Doppler velocimetry data of turbulent flows in rotating and reciprocating machinery. Exp Fluids 29(5):411-417. doi:10.1007/s003489900106

Graftieaux L, Michard M, Nathalie G (2001) Combining PIV, POD and vortex identification algorithms for the study of unsteady turbulent swirling flows. Meas Sci Technol 12(9):1422-1429

Griffiths A, Yazdabadi P, Syred N (1998) Alternate eddy shedding set up by the nonaxisymmetric recirculation zone at the exhaust of a cyclone dust separator. J Fluids Eng Trans ASME 120(1):193-199

Guggenberger M, Senn F, Schiffer J, Jaberg H, Gentner C, Sallaberger M, Widmer C (2014) Experimental investigation of the turbine instability of a pump-turbine during synchronization. IOP conference series: earth and environmental science, 22. doi: $10.1088 / 1755-1315 / 22 / 3 / 032015$

Gupta AK, Lilley DG, Syred N (1984) Swirl flows, vol 1. Abacus Press, UK

IEC Standards (1999) 60193: hydraulic turbines, storage pumps and pump-turbines - model acceptance tests, 2nd edn. International Commission

Iliescu M, Ciocan G, Avellan F (2008) Analysis of the cavitating draft tube vortex in a francis turbine using particle image velocimetry measurements in two-phase flow. J Fluids Eng Trans ASME 130(2):021105

Ingvorsen K, Meyer K, Walther J, Mayer S (2013) Turbulent swirling flow in a model of a uniflow-scavenged two-stroke engine. Exp Fluids 54(3): 1-7

Jeong J, Hussain F (1995) On the identification of a vortex. J Fluid Mech 285:69-94

Kirschner O, Ruprecht A, Göde E, Riedelbauch S (2012) Experimental investigation of pressure fluctuations caused by a vortex rope in a draft tube. IOP conference series: earth and environmental science, 15

Kuibin P, Susan-Resiga R, Muntean S (2013) A model for precessing helical vortex in the turbine discharge cone. IOP conference series: earth and environmental science, 22

Landry C, Favrel A, Müller A, Nicolet C, Yamamoto K, Avellan F (2014) Experimental investigation of the local wave speed in a draft tube with cavitation vortex rope. IOP conference series: earth and environmental science, 22

Lucca-Negro O, O'Doherty T (2001) Vortex breakdown: a review. Prog Energy Combust Sci 27(4):431-481

Martinelli F, Cozzi F, Coghe A (2012) Phase-locked analysis of velocity fluctuations in a turbulent free swirling jet after vortex breakdown. Exp Fluids 53(2):437-449

Müller A, Dreyer M, Andreini N, Avellan F (2013) Draft tube discharge fluctuation during self-sustained pressure surge: fluorescent particle image velocimetry in two-phase flow. Exp Fluids 54(4):1-11 
Müller A, Yamamoto K, Alligné S, Yonezawa K, Tsujimoto Y, Avellan F (2016) Measurement of the self-oscillating vortex rope dynamics for hydroacoustic stability analysis. ASME J Fluids Eng 138(2). doi:10.1115/1.4031778

Nishi M, Liu S (2013) An outlook on the draft-tube-surge study. Int J Fluid Mach Syst 6(1):33-48

Nishi M, Matsunaga S, Kubota T, Senoo Y (1982) Study on swirl flow and surge in an elbow type draft tube. In: Proceedings of the 10th IAHR symposium, Tokyo, Japan, vol 1, pp 557-568

Rheingans W (1940) Power swings in hydroelectric power plants. Trans ASME 62:171-184

Susan-Resiga R, Ciocan G, Anton I, Avellan F (2006) Analysis of the swirling flow downstream a Francis turbine runner. J Fluids Eng Trans ASME 128(1):177-189
Syred N (2006) A review of oscillation mechanisms and the role of the precessing vortex core (PVC) in swirl combustion systems. Prog Energy Combust Sci 32(2):93-161

Takamura H, Ebara S, Hashizume H, Aizawa K, Yamano H (2012) Flow visualization and frequency characteristics of velocity fluctuations of complex turbulent flow in a short elbow piping under high reynolds number condition. J Fluids Eng Trans ASME 134(10): 101201

Widmer C, Staubli T, Lederberger N (2011) Unstable characteristics and rotating stall in turbine brake operation of pump-turbines. $\mathrm{J}$ Fluids Eng Trans ASME 133(4):041101 